\title{
Extracorporeal Membrane Oxygenation Bridge to Lung Transplantation in a Patient with Hermansky- Pudlak Syndrome and Progressive Pulmonary Fibrosis
}

\author{
Wooho Sim', Song Yee Kim ${ }^{1}$, Jinu Han², Tyler Hyungtaek Rim², Jin Gu Lee ${ }^{3}$, Hyo Chae Paik ${ }^{3}$, Moo Suk Park ${ }^{1}$ \\ ${ }^{1}$ Division of Pulmonology, Department of Internal Medicine, Institute of Chest Diseases, ${ }^{2}$ Department of Ophthalmology, Institute of Vision Research, and \\ ${ }^{3}$ Department of Thoracic and Cardiovascular Surgery, Severance Hospital, Yonsei University College of Medicine, Seoul, Korea
}

\section{Dear Editor:}

Hermansky-Pudlak syndrome (HPS) is a group of rare, heterogeneously inherited, autosomal recessive disorders, presenting with oculocutaneous albinism, bleeding diathesis, and pulmonary disease. Pulmonary fibrosis is one of the fatal systemic manifestations of HPS [1,2]. Lung transplantation may be the only treatment option for patients with HPS, who have severe pulmonary fibrosis. There are several reports of patients who have undergone lung transplantation, despite bleeding diathesis observed in HPS. El-Chemaly et al. [3] have reported six patients who were evaluated for lung transplantation, and three of the patients survived after receiving lung transplantation. Umei et al. [4] have reported the successful use of venovenous extracorporeal membrane oxygenation (VV-ECMO) as a bridge in a patient with HPS associated with severe pulmonary fibrosis.

This study was approved by the Institutional Review Board of our institution (IRB No. 4-2013-0770) and informed consent from the patient for lung transplantation study was obtained. In this report, we describe a patient with HPS, who underwent bilateral lung transplantation after ECMO bridge treatment in Korea. A 57-year-old female patient was referred to our hospital to be evaluated for lung transplantation as a treatment option because of an increasing requirement for supplementary oxygen. She had been followed up as an outpatient after having been diagnosed with interstitial lung disease, based on computed tomography of the lungs that was performed 9 months before. She had started home oxygen treatment 6 months before. Her younger sister had died because of severe pulmonary fibrosis a week before the patient's first visit to our hospital. Her younger brother, who had been diagnosed with typical interstitial pneumonia by lung biopsy, had died 6 years before. The patient's father, younger brother, and younger sister had a history of heart disease.

On the physical examination, the patient was alert and oriented. She had hyperkeratinized eczematous skin lesions on the face and both arms, hypopigmentation of the iris, and lightcolored body hair (Figure 1). She mentioned that her daughter also had a light-colored skin and body hair. Her blood pressure was in the normal range, but her heart rate was elevated up to 110 beats per minute, and the respiratory rate was rapid ( 25 breaths per minute). The patient needed wheelchair ambulation and oxygen supplementation via a rebreathing mask ( $4 \mathrm{~L} / \mathrm{min}$ ). The forced vital capacity was $28 \%$ of the predicted value. On imaging examinations, posteroanterior chest radiograph and computed tomography scans showed progressing bi-

\section{Letter to the Editor}

Received: December 6, 2018

Revised: February 10, 2019

Accepted: February 13, 2019

Corresponding author Moo Suk Park

Division of Pulmonology, Department of Internal Medicine, Institute of Chest Diseases, Severance Hospital, Yonsei University College of Medicine, 50-1 Yonsei-ro, Seodaemun-gu, Seoul 03722, Korea Tel: +82-2-2228-1955 Fax: +82-2-393-6884 E-mail:pms70@yuhs.ac

Copyright (@) 2019 The Korean Society of Critical Care Medicine

This is an Open Access article distributed under the terms of Creative Attributions Non-Commercial License (http:// creativecommons.org/li-censes/by-nc/4.0/ which permits unrestricted noncommercial use, distribution, and reproduction in any medium, provided the original work is properly cited. 
lateral pulmonary fibrosis (Figure 2).

We planned to discharge the patient after evaluation for lung transplantation; however, the supplemental oxygen demand of the patient rapidly increased during the admission period. Finally, we transferred the patient to the intensive care unit and started a high-flow oxygen treatment on the 24th day of hospitalization.

On the 3rd day in the intensive care unit, we intubated the patient and started mechanical ventilation and VV-ECMO because she was not able to tolerate the high-flow oxygen treatment. Meanwhile, we were waiting for the results of genetic examination, which was being conducted because of the possibility of HPS, based on the patient's clinical features and family history. Although we did not have a confirmative diagnosis of HPS, we had to be cautious about the potential bleed-
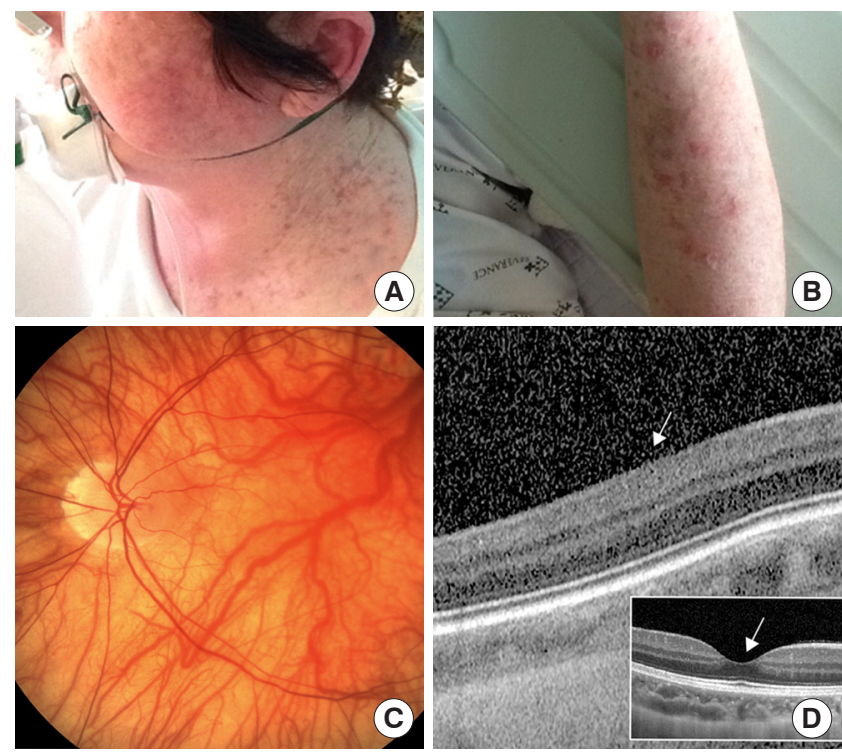

Figure 1. (A, B) Hyperkeratinized eczematous skin lesions were seen on the face and both arms. (C) Fundus photograph shows a depigmented retina. (D) Spectralis optical coherence tomography revealed absence of central pit, which was consistent with fovea plana (arrow: absence of foveal pit), whereas normal control showed normal foveal pit in small white box (arrow). ing tendency. We conducted a platelet multi-function test prior to commencing VV-ECMO and found that the results were in the normal range.

On the 4th day of ECMO, the patient showed changes in the skin color of both lower extremities, which was suspected to be due to heparin-induced thrombocytopenia or thrombophlebitis. We continued the heparin infusion to maintain the activated clotting time in the range between 150 and $170 \mathrm{sec}$ onds, while closely observing the condition of the patient's skin. On the 7th day of ECMO, we performed fiberoptic bronchoscopy because of bleeding from the endotracheal tube. We could not find a definite bleeding focus, except that there was a single bronchial ulcerative lesion, which was suspected to be a suction tip-caused injury. We consistently conducted mechanical ventilator weaning, and the final settings of the ventilator, just before transplantation, were pressure support ventilation with $10 \mathrm{mmHg}$ of pressure support, $50 \%$ fraction of inspired oxygen, and $7 \mathrm{mmHg}$ of continuous positive airway pressure. The patient maintained an alert mental status from the 3rd day after starting ECMO until the day of transplantation, without infusion of sedatives.

We conducted bilateral lung transplantation after changing the ECMO configuration to the veno-arterial system on the 10th day after VV-ECMO insertion. The operation was completed without severe complications, and the patient was weaned from ECMO in the operation room and transferred to the intensive care unit. Histopathology of the explanted lungs showed marked diffuse fibrosis, indicating chronic fibrosing interstitial pneumonia.

Genetic examination confirmed HPS 3 days after lung transplantation. The data showed p.Leu28Ter and p.Glu666Lys heterozygous variants in the HPS1 gene, confirming the diagnosis of HPS (Table 1).

The patient was transferred to a general ward 12 days after transplantation when she was able to tolerate oxygen administration through a tracheostomy mask. The patient was discharged from the hospital on the 45th day after transplanta-

Table 1. Results of the genetic examination of HPS

\begin{tabular}{|c|c|c|c|c|c|c|c|}
\hline Gene & Nucleotide & Amino acid & Zygosity & SIFT & PolyPhen-2 & CADD & gnomAD \\
\hline HPS1 & c.81delG & p.Leu28Ter & Heterozygote & - & - & $24.8^{\mathrm{a}}$ & Not found \\
\hline HPS1 & c. $1996 G>A$ & p.Glu666Lys & Heterozygote & deleterious $(0)^{b}$ & $\begin{array}{l}\text { probably damag- } \\
\text { ing }(0.999)^{c}\end{array}$ & $31^{\mathrm{a}}$ & $2 / 248814^{d}$ \\
\hline
\end{tabular}

HPS: Hermansky-Pudlak syndrome; SIFT: sorting intolerant from tolerant; PolyPhen-2: polymorphism phenotyping v2; CADD: combined annotationdependent depletion; gnomAD: genome aggregation database.

${ }^{a}$ CADD score of greater of equal 10 indicates that these are predicted to be the $10 \%$ most deleterious substitutions, a score of greater or equal 20 indicates the $1 \%$ most deleterious and so on; ${ }^{b}$ Probabilities less than 0.05 are predicted to be deleterious; ${ }^{c}$ Probabilistic score above 0.85 is classified as

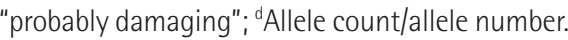



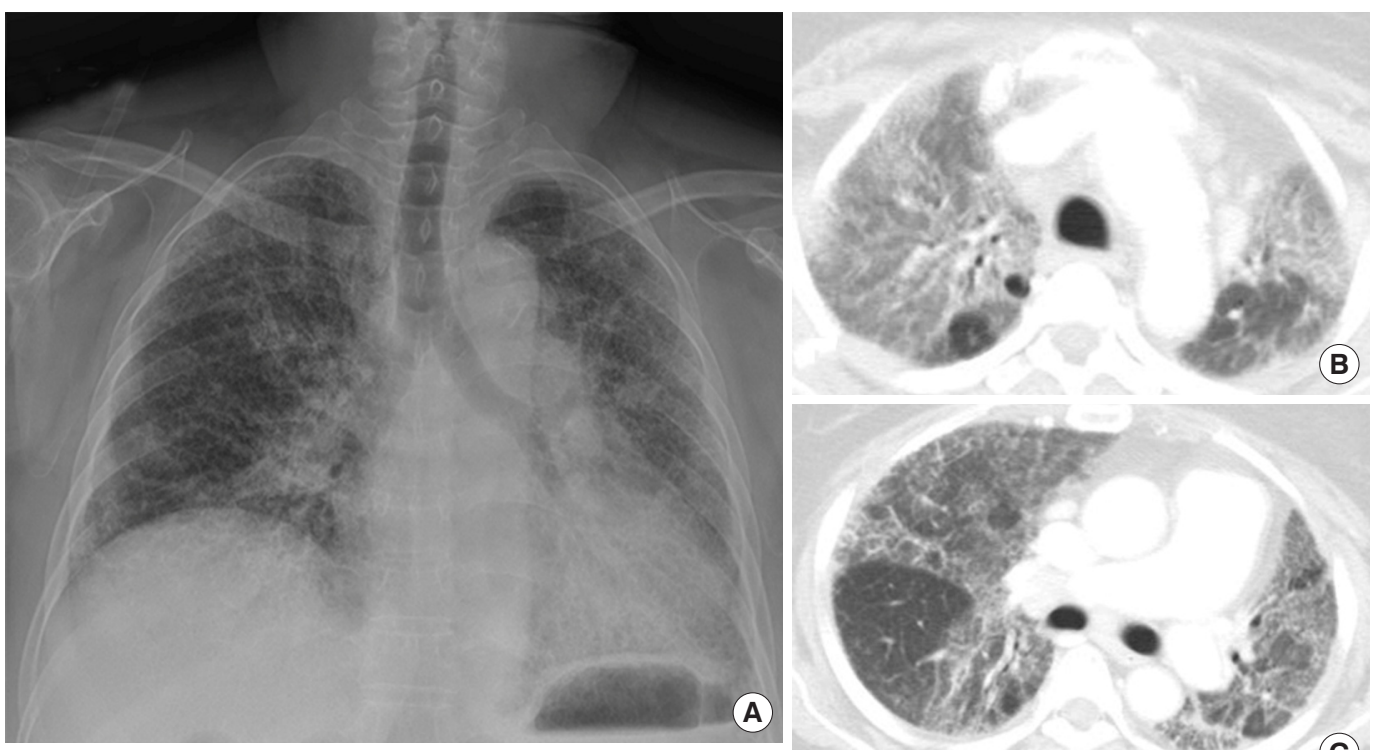

Figure 2. (A) Posteroanterior chest radiograph taken before lung transplantation shows peribronchial ground-glass opacity and reticulation in both lung zones. (B-D) Contrast-enhanced computed tomography scans show multifocal peribronchial ground-glass opacity with traction bronchiectasis and reticulation, indicating bilateral pulmonary fibrosis.
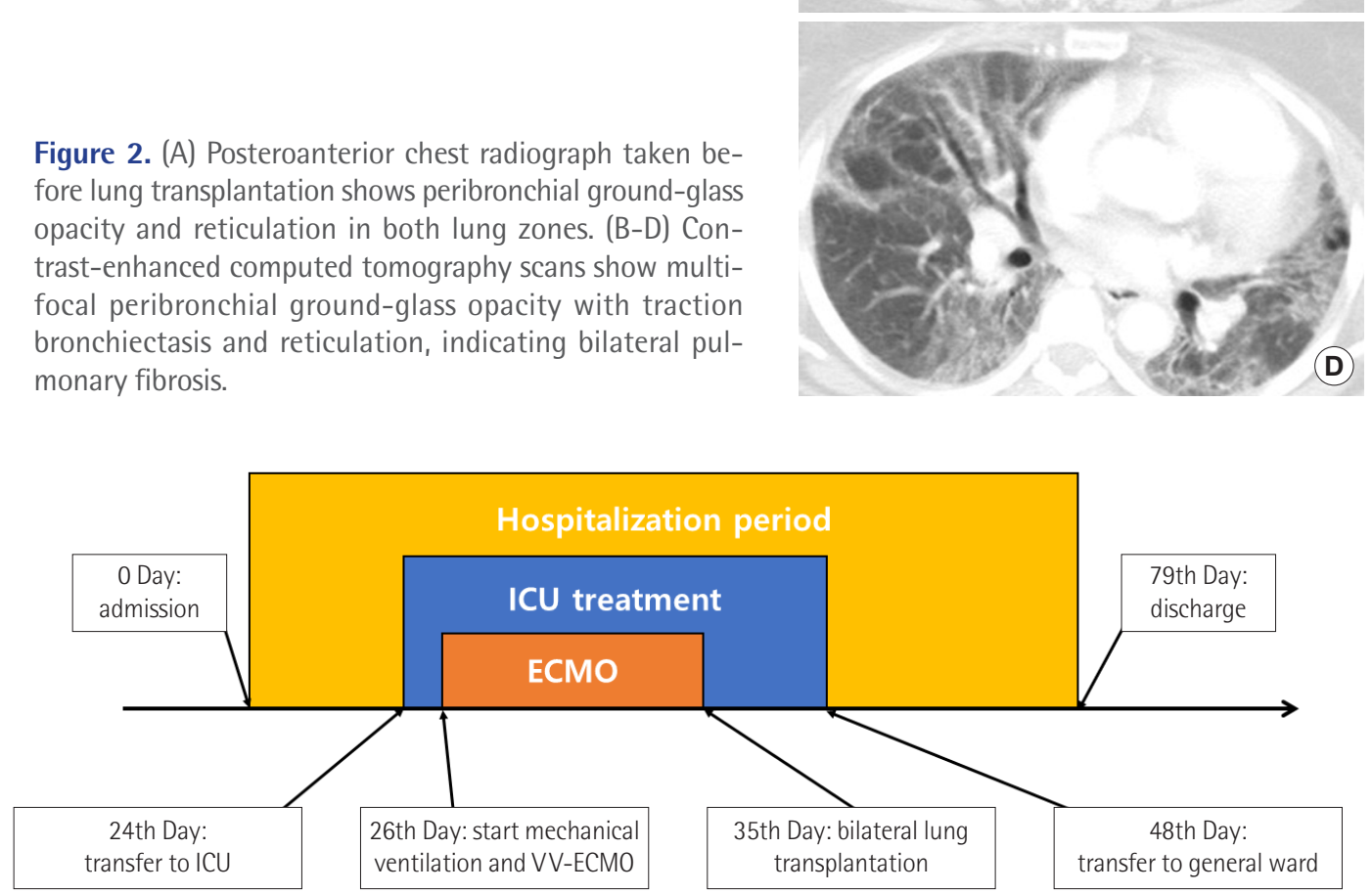

Figure 3. Diagram of the first hospitalization period. ICU: intensive care unit; ECMO: extracorporeal membrane oxygenation; W-ECMO: veno-venous ECMO.

tion (Figure 3).

The patient visited the emergency room approximately 20 days after discharge because of a sudden aggravation of dyspnea. We detected newly developed pneumonia and a pleural effusion. We started intravenous antibiotics and inserted a percutaneous catheter drainage tube to the left thorax. Furthermore, we conducted bronchial ballooning of the narrowed left bronchus and stent insertion in the left pulmonary artery on account of stenosis and a mismatch in the ventilation to perfusion ratio. The patient was subsequently discharged 100 days after the second admission. We have now been following her up for more than 11 months as an outpatient.

To our knowledge, this is the first case report of a patient with HPS, who underwent bilateral lung transplantation in Korea. Moreover, we performed VV-ECMO as a bridge to lung transplantation without significant complications, despite the risk of increased bleeding tendencies in patients with HPS. 


\section{CONFLICT OF INTEREST}

No potential conflict of interest relevant to this article was reported.

\section{ORCID}

Wooho Sim https://orcid.org/0000-0002-3417-1364

Song Yee Kim https://orcid.org/0000-0001-8627-486X

Jinu Han https://orcid.org/0000-0002-8607-6625

Tyler Hyungtaek Rim https://orcid.org/0000-0001-6465-2620 Jin Gu Lee https://orcid.org/0000-0003-2767-6505

Hyo Chae Paik https://orcid.org/0000-0001-9309-8235

Moo Suk Park

\section{AUTHOR CONTRIBUTIONS}

Conceptualization: WS, MSP. Data curation: WS, SYK. Formal analysis: WS, JH, THR. Methodology: WS, MSP. Visualization: JH, THR. Writing - original draft: WS, MSP. Writing - review \& editing: all.

\section{REFERENCES}

1. Huizing M, Malicdan MC, Gochuico BR, Gahl WA. Hermansky-Pudlak syndrome [Internet]. Seattle (WA): University of Washington; 1993-2017 [cited 2019 Feb 25]. Available from: https://www.ncbi.nlm.nih.gov/books/NBK1287/.

2. Vicary GW, Vergne Y, Santiago-Cornier A, Young LR, Roman J. pulmonary fibrosis in Hermansky-Pudlak syndrome. Ann Am Thorac Soc 2016;13:1839-46.

3. El-Chemaly S, O'Brien KJ, Nathan SD, Weinhouse GL, Goldberg HJ, Connors JM, et al. Clinical management and outcomes of patients with Hermansky-Pudlak syndrome pulmonary fibrosis evaluated for lung transplantation. PLoS One 2018;13: e0194193.

4. Umei N, Ichiba S, Chida M. Successful use of veno-venous extracorporeal membrane oxygenation as a bridge to lung $\mathrm{T}$ transplantation in a patient with pulmonary fibrosis. Gen Thorac Cardiovasc Surg 2017;65:478-80. 